7. Livshiz-Riven I, Nativ R, Borer A, Kanat-Maymon Y, Anson O. Nursing students' intentions to comply with standard precautions: an exploratory prospective cohort study. Am J Infect Control 2014;42:744-749.

8. Pontivivo G, Rivas K, Gallard J, Yu N, Perry L. A new approach to improving hand hygiene practice in an inner city acute hospital in Australia. Healthcare Infect 2012;17:57-63.

9. Charani E, Ahmad R, Rawson TM, Castro-Sanchez E, Tarrant C, Holmes AH. The differences in antibiotic decision-making between acute surgical and acute medical teams: an ethnographic study of culture and team dynamics. Clin Infect Dis 2019;69:12-20.

10. Elango S, Szymczak JE, Bennett IM, Beidas RS, Werner RM. Changing antibiotic prescribing in a primary care network: the role of readiness to change and group dynamics in success. Am J Med Qual 2018;33: $154-161$.

\title{
Food service: An overlooked potential vector for hospital infection
}

\author{
John D. Carter Jr (1)
}

Carter Exterminating Company, Cleveland, Ohio

To the Editor-In more than 25 years of working with hospitals to eliminate pests, I have observed an increasingly superficial approach to reducing the sources of sustenance for insects ${ }^{1}$ and rodents ${ }^{2}$ that can serve as vectors for disease-causing organisms. Conditions have degenerated from the time when hospitals had dedicated food service professionals as employees. Now this work commonly is done by contractors without the same knowledge, commitment, ${ }^{3}$ or hospital-specific training.

Hospitals largely rely on topical agents for cleaning that address the surface contaminants but not the residual contamination. Instead of eliminating the hidden food-decaying organisms that attracts flies, hospitals now use electrocution devices that introduce and disseminate exploded insect bodies into HVAC systems, further contaminating surgical theatres, ICUs, and patient rooms. Figure 1 provides examples of the many potentially diseasetransmitting conditions I have observed.

Certain species of filth flies can travel for miles within a 24-hour period searching an entire facility for decaying organic matter for additional propagation. ${ }^{4}$ Adult fruit, drain, or moth flies are prolific breeders, resulting in a wide area of invasion far from the original breeding sites. Therefore, all occurrences of degenerating organic debris can be original or satellite breeding opportunities.

Filth flies are capable of breeding in more types of materials than any other structure-infesting fly. In buildings, they be may found breeding in drains, trash containers, dumpsters, rotting produce, recycle bins, grease traps, garbage disposals, crawl spaces, and any site where moist organic matter can accumulate for a few days or longer. Fly larvae have been found in the open wounds of patients, and even in the mouths of intubated patients (I have personally seen this).

Food service areas present the number-one opportunity to prevent disease. Food contaminates! It attracts insects and other pests. We need to understand this potential for contamination when storing and preparing food and utensils. Hospital buildings are like our own human bodies. The very systems that support our lives also are vulnerabilities for infection. Bricks and mortar can protect the hospital like the integument protects the body. Heating ventilation and air conditioning (HVAC) systems can exclude or admit pathogens in the same way as the human respiratory system. Food-

Author for correspondence: John D. Carter, E-mail: john@carterexterminating.com Cite this article: Carter JD. (2020). Food service: An overlooked potential vector for hospital infection. Infection Control \& Hospital Epidemiology, 41: 630-631, https:// doi.org/10.1017/ice.2020.39 waste management systems can prevent or allow viruses or bacteria, just like the digestive system.

Pests are attracted to buildings in the same way that diseasecausing pests are attracted to humans. We invite them in with smells and opportunity. We give them a home in decaying matter. We use a symptom-based approach instead of a thorough evaluation and investigation. When we do not appreciate and address these attractions, we end up with pests of all sorts and increased potential for disease.

Pesticide use is regulated and cannot be a substitution for sanitation and maintenance. In situations where organic debris are degenerating, topical pesticide applications degenerate much faster than their intended label application parameters, and anti-pest baits are mitigated by food competition.

A 3-word definition of food sanitation is protection from contamination. With this in mind, all hospital functions and operations must be included in a sanitation program. All food products must be protected from contamination from the receiving dock (and before), right through distribution and removal. Sanitation is a dynamic and ongoing function and cannot be sporadic. When embedded in a healthcare facility with a special functions (eg, surgical theatre, pharmacy, dialyses care unit, etc), it becomes even more critical. Adequate cleaning and maintenance are crucial

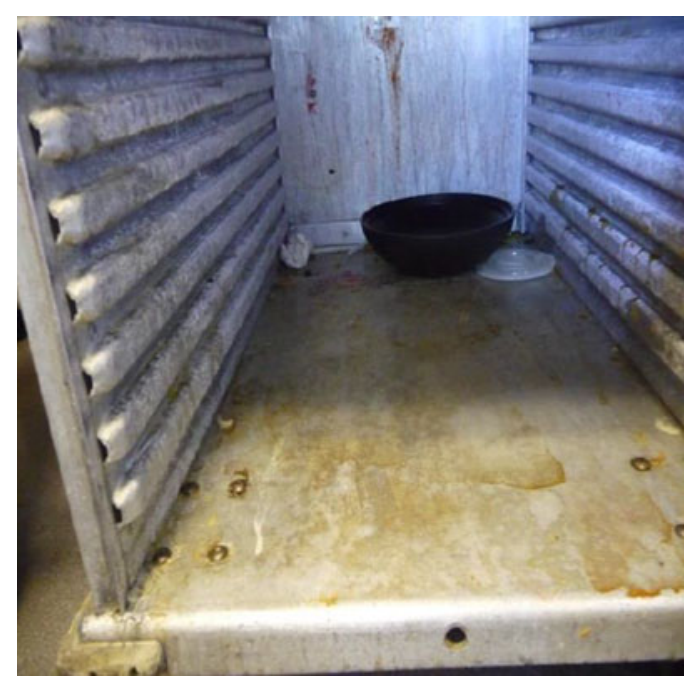

Fig. 1. Patient floor stationed food tray cart: satellite fly proliferation site. 
first steps in environmentally compatible fly control and will provide long-term discouragement of additional pest invaders.

I recommend the following systematic approach: (1) prioritized leadership and hospital administrative commitment to sanitation; (2) empowering and educating food service workers in illness prevention; (3) emphasizing environmentally compatible sanitation methods coupled with strategic and specific applications of antipest agents when required; and (4) looking beyond surface cleanliness to follow food as an attractor of pests.

Acknowledgments. Kurt C. Stange, $\mathrm{MD}, \mathrm{PhD}$, provided helpful guidance in writing this commentary.

Financial support. No financial support was provided relevant to this article.
Conflicts of interest. The author reports no conflicts of interest relevant to this article.

\section{References}

1. Khamesipour F, Lankarani KB, Honarvar B, Kwenti TE. A systematic review of human pathogens carried by the housefly (Musca domestica L.). BMC Public Health 2018;18:1049.

2. Grantz, N. Vector-and Rodent-Borne Diseases in Europe and North America. Cambridge, UK: Cambridge University Press, 2006.

3. Giraldo YM, Leitch KJ, Ros IG, et al. Sun navigation requires compass neurons in Drosophila. Curr Biol 2018;28:2845-2852.

4. Armed Forces Pest Management Board. Filth flies: significance and control in contingency operations. Office of the Under Secretary of Defense website. https://www.acq.osd.mil/eie/afpmb/docs/techguides/tg30.pdf. Published April 2002. Accessed December 28, 2019.

\title{
High minimum inhibitory concentrations among derepressed AmpC-beta-lactamase-producing Enterobacter cloacae complex isolates for ceftolozane with tazobactam
}

\author{
Leandro Reus Rodrigues Perez $\mathrm{PhD}^{1}$ (1), Eliana Carniel BSc ${ }^{2}$ and Gabriel Azambuja Narvaez MD ${ }^{3}$ \\ ${ }^{1}$ Universidade Federal de Ciências da Saúde de Porto Alegre, Porto Alegre, State of Rio Grande do Sul, Brazil, ${ }^{2}$ Universidade Feevale, Novo Hamburgo, State of \\ Rio Grande do Sul, Brazil and ${ }^{3}$ Hospital Mãe de Deus, Porto Alegre, State of Rio Grande do Sul, Brazil
}

To the Editor-Enterobacterales, such as Enterobacter spp, Serratia marcescens, Citrobacter freundii, Providencia spp and Morganella morganii, often referred to as the ESCPM group, may express high levels of chromosomal AmpC $\beta$-lactamases at high levels following exposure to $\beta$-lactams, mainly after third-generation cephalosporin therapy. ${ }^{1}$ The induction or selection of derepressed isolates is a concern because they contribute to the isolation of organisms no longer susceptible to specific $\beta$-lactams and may lead to clinical failure, with scarce antimicrobial options. ${ }^{2}$

Ceftolozane with tazobactam $(\mathrm{C} / \mathrm{T})$ is a combination drug comprising a $\beta$-lactamase inhibitor (tazobactam) with a new cephalosporin (ceftolozane). Tazobactam inhibits class A extendedspectrum $\beta$-lactamases (EBSLs), and ceftolozane acts via a high affinity for some penicillin-binding-protein (PBPs). C/T is stable in the presence of AmpC $\beta$-lactamases and against OprD deficiency and efflux pumps. These characteristics make the $\mathrm{C} / \mathrm{T}$ combination an important weapon in the treatment of infections due to extensively resistant $P$ seudomonas aeruginosa that are not carbapenemase producers. ${ }^{3}$

Despite the high efficacy described so far, emergence of resistance to $\mathrm{C} / \mathrm{T}$, mainly in $P$. aeruginosa isolates overexpressing AmpC- $\beta$-lactamase enzymes, have been reported. ${ }^{4}$ Although derepressed AmpC may occur in P. aeruginosa, the main target for $\mathrm{C} / \mathrm{T}$ use, this resistance mechanism is more robust in Enterobacter cloacae complex isolates, with a higher ability than others from

Author for correspondence: Leandro Reus Rodrigues Perez, E-mail: leandro.reus@ gmail.com

Cite this article: Perez LRR, Carniel E, and Narvaez GA. (2020). High minimum inhibitory concentrations among derepressed AmpC-beta-lactamase-producing Enterobacter cloacae complex isolates for ceftolozane with tazobactam. Infection Control \& Hospital Epidemiology, 41: 631-633, https://doi.org/10.1017/ice.2020.31 the ESCPM group to derepress AmpC- $\beta$-lactamase production, which has important clinical and therapeutic implications. ${ }^{5}$

The main objective of this study was to determine the $\mathrm{C} / \mathrm{T}$ minimum inhibitory concentration (MIC) among E. cloacae complex isolates, producing or not derepressed AmpC- $\beta$-lactamases. Additionally, meropenem and ceftazidime/avibactam MICs were also determined.

A set of 123 E. cloacae complex isolates recovered from inpatients between August 2016 and December 2017, in southern Brazil, were included in this study. Bacterial identification was made using the Vitek 2 automated system (bioMérieux, Marcy I'Etoile, France) and matrix-assisted laser desorption/ ionization time-of-flight mass spectroscopy (MALDI-TOF MS) for confirmation when necessary. Ertapenem susceptibility was determined using disc-diffusion testing. ${ }^{6}$ The MICs of cefotolozane/ tazobactam, meropenem, and ceftazidime/avibactam were determined using MIC test strips (MTS, Liofilchem, Italy) and were interpreted according to European Committee on Antimicrobial Susceptibility Testing (EUCAST) break points. ${ }^{6}$ To attribute the resistance mechanism for the selected E. cloacae complex isolates, a synergistic test was applied using an enzymatic inhibition testing with clavulanic acid and cloxacillin and/or phenyl-boronic acid to detect ESBLs and AmpC enzymes, in that order, as reported elsewhere. ${ }^{7}$ No isolate with carbapenemase production was included in this study, and for this study, all isolates were screened for a negative result using a blue-carba test to exclude class A and B carbapenemases and an OKN K-set immunochromatographic assay to exclude OXA-48-like production (ie, a carbapenemase with low hydrolysis activity for carbapenems and eventually resulting in a negative blue-carba test). ${ }^{8}$

Among the 123 isolates, 39 (31.7\%) and 84 (68.3\%) were characterized as derepressed and not-derepressed AmpC- $\beta$-lactamase 